\title{
Adjuvantes nas propriedades físicas da calda, espectro e eficiência de eletrificação das gotas utilizando a pulverização eletrostática
}

\author{
Adjuvant on spray solution physical properties droplets spectrum and electrification efficiency \\ using electrostatic spraying
}

\author{
Robson Shigueaki Sasaki ${ }^{I}$ Mauri Martins Teixeira ${ }^{\text {II }}$ Humberto Santiago ${ }^{\text {III }}$ \\ Ronaldo Porto Madureira ${ }^{\text {III }}$ Christiam Felipe Silva Maciel $^{\text {III }}$ \\ Haroldo Carlos Fernandes ${ }^{\text {II }}$
}

RESUMO

\begin{abstract}
Os adjuvantes têm sido empregados na pulverização de agrotóxicos, entretanto, ainda se carece de informações sobre $o$ efeito destes produtos, principalmente quando se emprega $o$ sistema eletrostático. $O$ presente trabalho foi conduzido com o objetivo de avaliar dez soluções de pulverização (nove adjuvantes e uma testemunha), quanto às propriedades físicas (viscosidade e condutividade elétrica) e quanto ao espectro e a eficiência de eletrificação da gota na pulverização eletrostática. Utilizou-se um pulverizador eletrostático da marca Electrostatic Spraying Systems e modelo MB 4.0. Para avaliar a viscosidade, a metodologia de Ostwald foi utilizada, enquanto a condutividade elétrica foi determinada com uso de um condutivímetro, marca Marte e modelo MB-11. Na análise da carga elétrica presente na gota, utilizou-se a metodologia da gaiola de Faraday e a avaliação do espectro foi realizada por meio de um analisador de partículas a laser (Spraytech Malvern ${ }^{\circledR}$ ). Os adjuvantes testados alteraram a viscosidade da calda, variando entre 0,94 e 1,03mPa s. Para a condutividade elétrica, observou-se que a água apresentou $67,7 \mu \mathrm{S}$ $\mathrm{cm}^{-1}$ e, com o uso de adjuvantes, o valor obtido foi de até $607,5 \mu \mathrm{S}$ $\mathrm{cm}^{-1}$. Quanto à eletrificação das gotas, houve aumento de até $50,14 \%$ na relação $Q / M$ com o uso de adjuvantes, demonstrando que auxiliam na pulverização eletrostática. De forma geral, os adjuvantes reduziram o diâmetro das gotas pulverizadas.
\end{abstract}

Palavras-chave: tecnologia de aplicação de agrotóxicos, relação carga/massa, tamanho de gotas.

\section{ABSTRACT}

Adjuvants have been used in pesticide spraying, however, there is a need of further information about the effect of these products, especially when employing the electrostatic system. Thus, the aim of this study was to evaluate ten spray solution (nine adjuvants + control) concerning on physical properties (viscosity and electrical conductivity) and concerning on droplet spectrum and efficiency of droplet electrification in electrostatic spraying. The electrostatic sprayer Electrostatic Sraying Systems, MB 4.0 model, was used for this experiment. The Ostwald methodology was used to evaluate the viscosity, while the electrical conductivity was measured using a conductivity meter Marte, MB-11 model. The Faraday cage was used for the analysis of electric charges in the droplets and the droplets spectrum was obtained by the particle size analyzer Malvern Spraytech. Adjuvants tested altered the viscosity of the spray solution between 0.94 and $1.03 \mathrm{mPa}$ s. For the electrical conductivity it was observed that the water had the mean conductivity of $67.7 \mu \mathrm{S} \mathrm{cm} \mathrm{cm}^{-1}$, and depending on the adjuvant type it was obtained values up to $607.5 \mu \mathrm{S} \mathrm{cm}^{-1}$. For the droplets electrification, there was an increasing up to $50.14 \%$ in the $C / M$ ratio by using the adjuvants, demonstrating that adjuvants may assist the electrostatic spraying. Generally, adjuvants reduced the droplet diameter.

Key words: technology pesticide application, charge/mass ratio, droplets size.

\section{INTRODUÇÃO}

Algumas tecnologias têm sido pesquisadas e incorporadas à pulverização, visando a auxiliar na aplicação correta de defensivos agrícolas, dentre elas tem se ressaltado o uso dos adjuvantes agrícolas. Os adjuvantes são compostos, adicionados às formulações ou à calda de pulverização, trazendo benefícios como aumento no molhamento, na aderência, no espalhamento, na redução de espuma

IDepartamento de Engenharia e Computação (DEC), Instituto Federal Minas Gerais (IFMG), 38900-000, Bambuí, MG, Brasil. E-mail: robson.sasaki@ifmg.edu.br. Autor para correspondência.

IIDepartamento de Engenharia Agrícola (DEA), Universidade Federal de Viçosa (UFV), Viçosa, MG, Brasil.

IIIPrograma de Pós-graduação em Engenharia Agrícola, UFV, Viçosa, MG, Brasil. 
e na dispersão da calda de pulverização (CUNHA \& PERES, 2010).

Ao adicionarem adjuvantes à calda, YU et al. (2009) constataram o efeito do espalhamento da gota ao se depositar no alvo. CUNHA et al. (2010a) relatam que a formação das gotas pode ser significativamente alterada, visto que estes produtos alteram as propriedades físicas das caldas, como a tensão superficial e a viscosidade. CUNHA \& ALVES (2009) avaliaram diferentes soluções aquosas e verificaram que, dependendo do tipo de produto, houve redução do $\mathrm{pH}$ e aumento da condutividade elétrica da solução. Porém, CUNHA et al. (2010b) observaram pouca variação quanto aos parâmetros pH e condutividade elétrica com o uso de adjuvantes. Ainda de acordo com CUNHA et al. (2010b) e CUNHA \& ALVES (2009), foi verificada a tendência em aumentar a viscosidade e reduzir a tensão superficial do líquido, empregando-se os adjuvantes.

Ao alterar as propriedades físicas da calda, pode também ocorrer alterações dos parâmetros técnicos da pulverização. MOTA \& ANTUNIASSI (2013) verificaram que o uso de adjuvantes elevou o diâmetro da mediana volumétrica (DMV) durante a pulverização. Entretanto, CUNHA et al. (2010b), avaliando o tamanho de gotas com a adição de adjuvantes à calda, constataram redução no tamanho das gotas pulverizadas, no entanto, BUENO et al. (2011) observaram que não houve alteração do parâmetro DMV, com o uso de adjuvantes.

Existem divergências quanto aos resultados obtidos em relação ao uso dos adjuvantes. Isto se deve ao fato de ocorrer interação entre as diferentes caldas de pulverização e à técnica empregada. Verifica-se ainda que os estudos estão concentrados na pulverização hidráulica, havendo poucas informações a respeito do uso de adjuvantes aliados à pulverização eletrostática. A pulverização eletrostática é um sistema que carrega eletricamente as gotas. A diferença de potencial elétrico entre a gota e o alvo faz com que as gotas sejam atraídas pela planta. Diversos trabalhos têm demonstrado a vantagem do uso do sistema eletrostático, como o realizado por SASAKI et al. (2013), na cultura do café, os quais verificaram que o sistema eletrostático aumentou a deposição de líquido em 37\%. ZHOU et al. (2012) relatam que o sistema eletrostático pode aumentar a deposição de agrotóxicos em até $60 \%$, além de reduzir as perdas em até $50 \%$ e reduzir os custos em até $20 \%$, quando comparado a outras técnicas de pulverização.

A eficiência do sistema eletrostático é altamente dependente das características do líquido pulverizado. As características do líquido pulverizado, como a tensão superficial e a viscosidade, podem interferir na pulverização eletrostática (ZHENG et al., 2002). MASKI \& DURAIRAJ (2010) afirmam que a condutividade elétrica e a constante dielétrica são as duas principais propriedades elétricas que afetam grandemente a carga adquirida pelas gotas pulverizadas. Ou seja, com a adição de adjuvantes à calda, ao se alterar as propriedades do líquido, pode-se aumentar ou reduzir a eficiência do sistema eletrostático.

Nesse contexto, o objetivo deste trabalho foi avaliar o efeito da adição de nove adjuvantes à calda, quanto às propriedades físicas do líquido, à eficiência de eletrificação e o espectro de gotas produzido na pulverização eletrostática.

\section{MATERIAL E MÉTODOS}

Avaliou-se o efeito de nove adjuvantes, mais a testemunha (água pura), quanto às propriedades físicas das soluções (viscosidade e condutividade elétrica), além dos parâmetros técnicos da pulverização eletrostática (relação carga/massa e espectro de gotas). A relação carga/massa $(\mathrm{Q} / \mathrm{M})$ é a quantidade de carga $(\mathrm{Q})$, em coulombs, por unidade de massa (M), em kg, presente nas gotas pulverizadas. Todos os ensaios foram realizados em condições de laboratório e conduzidos em delineamento inteiramente casualizado, com 10 tratamentos e quatro repetições.

Os produtos utilizados, o princípio ativo dos adjuvantes, bem como as doses utilizadas nos ensaios, comumente empregadas em campo, foram: 1) $\mathrm{Naft}^{\circledR}-105 \mathrm{~g} \mathrm{~L}^{-1}$ de $\mathrm{N}\left(0,05 \% \mathrm{v} \mathrm{v}^{-1}\right)$; 2) Silkon ${ }^{\circledR}$ $105 \mathrm{~g} \mathrm{~L}^{-1}$ de $\mathrm{N}$ e $52,5 \mathrm{~g} \mathrm{~L}^{-1}$ de $\left.\mathrm{P}_{2} \mathrm{O}_{5}\left(0,05 \% \mathrm{v} \mathrm{v}^{-1}\right) ; 3\right)$ TA $35^{\circledR}$ - Láuril Éter Sulfato Sódico $\left(0,05 \% \mathrm{v} \mathrm{v}^{-1}\right)$; 4$)$ Poliflex - 34,5g L-1 de N e $207 \mathrm{~g} \mathrm{~L}^{-1}$ de $\mathrm{P}_{2} \mathrm{O}_{5}(0,05 \% \mathrm{v}$ $\left.\left.\mathrm{v}^{-1}\right) ; 5\right)$ Silwet ${ }^{\mathbb{R}}-1000 \mathrm{~g} \mathrm{~L}^{-1}$ de Copolímero de poliéter e silicone $\left.\left(0,05 \% \mathrm{v} \mathrm{v}^{-1}\right) ; 6\right)$ Define ${ }^{\circledR}$ - Polímero Vegetal $\left.\left(0,06 \% v^{-1}\right) ; 7\right)$ Assist ${ }^{\circledR}-756 \mathrm{~g} \mathrm{~L}^{-1}$ de Óleo Mineral e $97 \mathrm{~g} \mathrm{~L}^{-1}$ de Ingredientes inertes $\left.\left(0,5 \% \mathrm{~V} \mathrm{v}^{-1}\right) ; 8\right)$ Aureo ${ }^{\circledR}$ - $720 \mathrm{~g} \mathrm{~L}^{-1}$ de Éster metílico de óleo de soja e $188 \mathrm{~g} \mathrm{~L}^{-1}$ de ingredientes inertes $\left(0,25 \% \mathrm{v} \mathrm{v}^{-1}\right)$; 9$)$ Agr'óleo $^{\circledR}$ $892 \mathrm{~g} \mathrm{~L}^{-1}$ de Éster de ácido graxo $\left(0,5 \% \mathrm{~V} \mathrm{v}^{-1}\right)$.

Para caracterizar as diferentes caldas quanto à viscosidade, utilizou-se o método de Ostwald. O viscosímetro empregado possui formato em "U", e é empregado submerso em solução com temperatura controlada. É dotado de um balão, dois bojos (superior e inferior) e duas marcações, de tal forma que o líquido é introduzido no balão e conduzido por sucção até atingir o bojo superior, 
onde, por efeito da gravidade, é escoado até o bojo inferior. O tempo que se leva para escoar entre os dois bojos determina a viscosidade do líquido. A temperatura durante a coleta de dados foi de $25^{\circ} \mathrm{C}$.

$\mathrm{Na}$ avaliação da condutividade elétrica, utilizou-se um condutivímetro, marca Marte e modelo MB-11, previamente aferido com a solução padrão. Durante este ensaio, as soluções foram monitoradas e permaneceram à temperatura de $25^{\circ} \mathrm{C}$.

Com as caldas previamente caracterizadas, realizaram-se os testes de relação carga/massa $(\mathrm{Q} / \mathrm{M})$ e espectro de gotas, utilizando-se um pulverizador eletrostático (modelo MBP 4.0, Electrostatic Spraying Systems, Watkinsville, GA, USA). O pulverizador é do tipo manual pneumático com eletrificação da gota pelo método indireto. O equipamento é constituído por um chassi do tipo carrinho de mão, um compressor de ar acionado por um motor ciclo Otto, quatro tempos e com potência de $4,85 \mathrm{~kW}$, mangueiras de ar e líquido, reservatório de calda com capacidade de $15,1 \mathrm{~L}$, e um circuito eletrônico ligado a duas baterias de 9V. Este pulverizador foi previamente regulado para operar na vazão de líquido de $0,150 \mathrm{~L} \mathrm{~min}^{-1}$.

Para determinar a intensidade de carga, utilizou-se o método da gaiola de Faraday isolada e ligada à terra por um multímetro, semelhantemente à metodologia empregada por MASKI \& DURAIRAJ (2010). As pulverizações foram realizadas à distância de $0,1 \mathrm{~m}$ do interior da gaiola, onde se realizaram as medições de corrente (A), com o auxílio de um multímetro, marca Minipa e modelo ET-2510, com precisão de $\pm 1 \%$. Neste ensaio, visando a garantir a eletrificação da gota e minimizar os erros, foi monitorada a tensão elétrica da bateria do pulverizador, de tal forma que sempre fosse mantida acima de 8,5V. Quando atingia valores abaixo desta tensão, era substituída.

Em todas as pulverizações, empregouse o pulverizador, com o motor operando à rotação de 3060rpm. Demarcou-se o tempo de 15 s até se estabilizar o equipamento e prosseguiu-se com as pulverizações. Posteriormente, com o auxílio de uma proveta graduada com precisão de $5 \mathrm{~mL}$, mensurou-se a quantidade de líquido pulverizada $\left(\mathrm{L} \mathrm{min}^{-1}\right)$. A partir dos dados da corrente elétrica e quantidade de líquido pulverizada, determinou-se a relação carga/massa presente na gota. Foi adotada a massa específica da água igual a um, para se obter o fluxo, em $\mathrm{kg} \mathrm{min}{ }^{-1}$. As pulverizações foram realizadas em condições de laboratório e todas as condições meteorológicas foram monitoradas durante o teste. Visando a minimizar os erros, cada repetição consistiu-se de 20 leituras no multímetro, perfazendo 80 leituras por tratamento, totalizando 800 leituras.
Quanto ao espectro de gotas, as avaliações foram realizadas com o auxílio de um analisador de partículas em tempo real (modelo Spraytech, Malvern Instruments Co, Malvern, GB-WOR, UK.), dotado de lente focal de $750 \mathrm{~mm}$ e precisão de $1 \%$. $\mathrm{O}$ equipamento foi configurado para mensurar gotas na faixa entre 0,1 a $2500 \mu \mathrm{m}$, com frequência de $2,5 \mathrm{kHz}$, e tempo de coleta de $1,5 \mathrm{~s}$, totalizando 3750 leituras, a cada pulverização realizada. As condições ambientais durante o experimento foram monitoradas. As pulverizações foram realizadas à distância de $0,15 \mathrm{~m}$ do laser. Os parâmetros da pulverização avaliados foram: $\mathrm{Dv}_{0,1} ; \mathrm{Dv}_{0,5} ; \mathrm{Dv}_{0,9}$; amplitude relativa (Span); $\% \mathrm{~V}<10 \mu \mathrm{m} ; 11<\% \mathrm{~V}<30 \mu \mathrm{m}$ e $31<\% \mathrm{~V}<50 \mu \mathrm{m}$. Nos ensaios de relação $\mathrm{Q} / \mathrm{M}$ e espectro de gotas, a temperatura da calda permaneceu entre 23 e $25^{\circ} \mathrm{C}$.

Os dados coletados foram tabulados em planilhas do Microsoft Excel ${ }^{\circledR}$, e as análises estatísticas foram realizadas com o auxílio do software Assistat v.7.6 beta. Anteriormente à análise de variância, foram verificados principalmente os pressupostos de homogeneidade de variância e normalidade dos erros, posteriormente, as médias foram comparadas pelo teste de Tukey a 5\% de probabilidade. Aplicouse também um teste de correlação de Pearson para verificar o grau de correlação entre a relação $Q / M$ e as demais variáveis.

\section{RESULTADOS E DISCUSSÃO}

$\mathrm{Na}$ avaliação das propriedades físicas das soluções, enquanto que a calda contendo somente água apresentou viscosidade de $1,00 \mathrm{mPa} \mathrm{s}$, ao adicionar os adjuvantes Define e Agr'óleo, houve aumento para $1,03 \mathrm{mPa}$ s. Com exceção do adjuvante Assist, os demais produtos tenderam a reduzir significativamente a viscosidade das caldas testadas. CUNHA et al. (2010b) verificaram alteração na viscosidade de diferentes caldas para a temperatura de $25^{\circ} \mathrm{C}$, variando entre 1,00 e $1,11 \mathrm{mPa} \mathrm{s}$, colaborando com o presente resultado. A viscosidade da calda em uma pulverização é de grande importância, pois pode-se alterar o diâmetro das gotas pulverizadas, e a modificação deste parâmetro, no caso da pulverização eletrostática, pode comprometer o carregamento delas, conforme constatado por CHAIM et al. (2002).

Quanto à condutividade elétrica, foi observada uma grande variação, de acordo com as diferentes soluções testadas. Enquanto que para a água a condutividade elétrica foi $67,74 \mu \mathrm{S} \mathrm{cm} \mathrm{cm}^{-1}$, para o produto Poliflex, foi de $607,5 \mu \mathrm{S} \mathrm{cm}^{-1}$. Com exceção dos produtos a base de Éster de ácido graxo e Copolímero de poliéter, todos os demais produtos 
elevaram os valores da condutividade elétrica da solução. Trabalhos realizados por CUNHA et al. (2010b) e CUNHA \& ALVES (2009) também verificaram esta tendência. Na avaliação da eficiência de eletrificação da gota, a temperatura e a umidade relativa do ar permaneceram entre 21 e $23,5^{\circ} \mathrm{C}$ e entre 75 e $91 \%$, respectivamente. Observou-se que o produto Poliflex apresentou maior condutividade elétrica e maior relação $\mathrm{Q} / \mathrm{M}$, com $5,45 \mathrm{mC} \mathrm{kg} \mathrm{kg}^{-1}$. Em contrapartida, o produto a base de Copolímero de poliéter apresentou a menor condutividade e relação Q/M, com 3,39 $\mathrm{mC} \mathrm{kg}^{-1}$ (Tabela 1). MASKI \& DURAIRAJ (2010), em um estudo com a pulverização eletrostática, atribuíram maior carga elétrica na gota a caldas com maior condutividade elétrica, colaborando com o presente resultado. Entretanto, afirmam que estudos relacionados a este fenômeno devem ser pesquisados.

É importante salientar que conhecer os parâmetros e/ou produtos que afetam a relação $\mathrm{Q} / \mathrm{M}$ na pulverização eletrostática é de grande interesse. Com exceção do Copolímero de poliéter, todos os demais adjuvantes tenderam a aumentar a relação $\mathrm{Q} / \mathrm{M}$, quando comparado à testemunha, demonstrando que podem aumentar a eficiência da pulverização eletrostática.

A eficiência da pulverização eletrostática depende diretamente da relação $\mathrm{Q} / \mathrm{M}$. Quanto maior esta relação, maior a força de atração entre a gota e o alvo, aumentando-se a qualidade da pulverização. De acordo com LAW (1995), a relação Q/M mínima é em torno de 1 a $2 \mathrm{mC} \mathrm{kg}^{-1}$. Em uma pulverização de campo, aplicar eficientemente significa dizer que, além de controlar as pragas, doenças ou plantas daninhas, as perdas para o meio ambiente são as mínimas possíveis, e sem deixar resíduos nos alimentos, sendo que a tecnologia eletrostática pode auxiliar neste quesito, conforme resultados obtidos por SASAKI et al. (2013). Além disso, o uso de adjuvantes, aliado ao sistema eletrostático, promete melhorar ainda mais a eficiência de aplicação, visto que houve a tendência de aumentar a relação $\mathrm{Q} / \mathrm{M}$.

$\mathrm{Na}$ análise do espectro de gotas, a temperatura do ar variou entre 20 e $22^{\circ} \mathrm{C}$ e a umidade relativa entre 81 e $85 \%$. A água pura apresentou valor de $\mathrm{DV}_{0,1}$ de $13,78 \mu \mathrm{m}$, enquanto que, com a adição do produto Define, esse valor foi reduzido para $7,64 \mu \mathrm{m}$. Quanto aos parâmetros $\mathrm{DV}_{0,5}$ e $\mathrm{DV}_{0,9}$, com exceção da calda contendo o princípio ativo de Copolímero de poliéter, todos os demais produtos reduziram significativamente estes parâmetros. Ao passo que para o Span, houve pequena alteração de acordo com os diferentes produtos testados (Tabela 2).

Foi observado que, com o uso de adjuvantes, houve a tendência de redução do diâmetro das gotas formadas, quando comparado à testemunha. Resultados opostos foram obtidos por MOTA \& ANTUNIASSI (2013), entretanto, tais autores empregaram a pulverização hidráulica. Isso mostra que o efeito da adição de um adjuvante não pode ser generalizado, pois existe interação com a ponta de pulverização (CUNHA, 2010b). No presente caso, além de utilizar o sistema de fragmentação de líquido pneumático, pode ter ocorrido a interação entre o método de carregamento e a geometria do eletrodo do sistema eletrostático, o que explica a redução no

Tabela 1 - Propriedades físicas e relação Q/M das diferentes soluções testadas.

\begin{tabular}{|c|c|c|c|c|c|c|}
\hline \multirow{2}{*}{$\begin{array}{l}\text { Produto } \\
\mathrm{H}_{2} \mathrm{O}\end{array}$} & \multicolumn{2}{|c|}{ Viscosidade ( $\mathrm{mPa} \mathrm{s})$} & \multicolumn{2}{|c|}{ Condutividade elétrica $\left(\mu \mathrm{S} \mathrm{cm}^{-1}\right)$} & \multicolumn{2}{|c|}{ Relação Q/M (mC kg $\left.{ }^{-1}\right)$} \\
\hline & 1,00 & $\mathrm{~b}$ & 67,74 & $\mathrm{~g}$ & 3,63 & $\mathrm{fg}$ \\
\hline Naft & 0,96 & de & 227,50 & $\mathrm{~b}$ & 4,88 & $\mathrm{~b}$ \\
\hline Silkon & 0,99 & $\mathrm{c}$ & 116,65 & $\mathrm{~d}$ & 4,8 & $\mathrm{bc}$ \\
\hline TA35 & 0,94 & $\mathrm{f}$ & 75,01 & $\mathrm{e}$ & 3,95 & def \\
\hline Poliflex & 0,96 & e & 607,50 & $\mathrm{a}$ & 5,45 & $\mathrm{a}$ \\
\hline Silwet & 0,97 & $\mathrm{~d}$ & 65,41 & $\mathrm{~g}$ & 3,39 & $\mathrm{~g}$ \\
\hline Define & 1,03 & $\mathrm{a}$ & 166,10 & $\mathrm{c}$ & 4,35 & $\mathrm{~cd}$ \\
\hline Assist & 0,99 & $\mathrm{~b}$ & 70,21 & $\mathrm{f}$ & 3,87 & ef \\
\hline Aureo & 0,98 & $\mathrm{c}$ & 76,31 & $\mathrm{e}$ & 4,19 & def \\
\hline Agr'óleo & 1,03 & $\mathrm{a}$ & 65,90 & $\mathrm{~g}$ & 3,93 & def \\
\hline$\rho$ de Pearson $(\mathrm{Q} / \mathrm{M})$ & $-0,28^{n}$ & & $0,79^{* *}$ & & 1,00 & \\
\hline DMS & 0,009 & & 1,948 & & 0,469 & \\
\hline $\mathrm{CV}(\%)$ & 0,39 & & 0,53 & & 4,57 & \\
\hline Média & 0,99 & & 337,15 & & 4,51 & \\
\hline
\end{tabular}

Médias seguidas por uma mesma letra na coluna não diferem entre si em nível de $5 \%$ de probabilidade pelo teste Tukey.* Significativo em nível de $1 \%$ de probabilidade pelo teste t. ${ }^{*}$ Significativo em nível de $5 \%$ de probabilidade pelo teste t. ${ }^{\text {ns }}$ Não significativo. 
Tabela 2 - Espectro de gotas com o uso de adjuvantes à calda.

\begin{tabular}{|c|c|c|c|c|c|c|c|}
\hline \multirow{2}{*}{ Produto } & \multicolumn{7}{|c|}{ Parâmetros $(\mu \mathrm{m})-$} \\
\hline & $\mathrm{DV}_{0,1}$ & $\mathrm{DV}_{0,5}$ & $\mathrm{DV}_{0,9}$ & Span(adm) & $\% \mathrm{~V}<10$ & $11<\% \mathrm{~V}<30$ & $31<\% \mathrm{~V}<50$ \\
\hline $\mathrm{H}_{2} \mathrm{O}$ & $13,8 \mathrm{a}$ & $31,0 \mathrm{a}$ & $58,5 \mathrm{a}$ & $1,4 \mathrm{ab}$ & $7,1 \mathrm{de}$ & $40,4 \mathrm{~d}$ & 33,9 a \\
\hline Naft & $8,7 \mathrm{~cd}$ & $21,9 \mathrm{~d}$ & $41,6 \mathrm{bc}$ & $1,5 \mathrm{ab}$ & $12,2 \mathrm{ab}$ & $58,7 \mathrm{ab}$ & $20,3 \mathrm{c}$ \\
\hline Silkon & $10,2 \mathrm{abcd}$ & $23,3 \mathrm{~cd}$ & $41,1 \mathrm{bc}$ & $1,3 \mathrm{ab}$ & 9,5 abcd & $59,1 \mathrm{ab}$ & $24,1 \mathrm{bc}$ \\
\hline TA35 & $13,2 \mathrm{ab}$ & $23,9 \mathrm{~cd}$ & $39,9 \mathrm{bc}$ & $1,1 \mathrm{~b}$ & $4,8 \mathrm{e}$ & $63,8 \mathrm{a}$ & $24,4 \mathrm{bc}$ \\
\hline Poliflex & $9,1 \mathrm{bcd}$ & $21,5 \mathrm{~d}$ & $38,8 \mathrm{c}$ & $1,4 \mathrm{ab}$ & $11,8 \mathrm{abc}$ & $60,8 \mathrm{ab}$ & $20,8 \mathrm{c}$ \\
\hline Silwet & $12,1 \mathrm{abc}$ & $28,6 \mathrm{ab}$ & $504 \mathrm{ab}$ & $1,3 \mathrm{ab}$ & 8,3 cde & $44,7 \mathrm{~cd}$ & $33,4 \mathrm{a}$ \\
\hline Define & $7,6 \mathrm{~d}$ & $24,2 \mathrm{~cd}$ & $46,0 \mathrm{bc}$ & $1,6 \mathrm{a}$ & $12,8 \mathrm{a}$ & $50,6 \mathrm{bcd}$ & $25,6 \mathrm{bc}$ \\
\hline Assist & 10,9 abcd & $25,6 \mathrm{bc}$ & $45,5 \mathrm{bc}$ & $1,3 \mathrm{ab}$ & $8,8 \mathrm{bcd}$ & $52,7 \mathrm{bc}$ & $28,4 \mathrm{ab}$ \\
\hline Aureo & 10,6 abcd & $22,7 \mathrm{~cd}$ & $40,9 \mathrm{bc}$ & $1,3 \mathrm{ab}$ & $8,9 \mathrm{abcd}$ & $60,9 \mathrm{ab}$ & $22,2 \mathrm{c}$ \\
\hline Agr'óleo & $12,3 \mathrm{abc}$ & $24,8 \mathrm{~cd}$ & $42,9 \mathrm{bc}$ & $1,2 \mathrm{ab}$ & $6,9 \mathrm{de}$ & $58,3 \mathrm{ab}$ & $26,1 \mathrm{bc}$ \\
\hline$\rho$ de Pearson $(\mathrm{Q} / \mathrm{M})$ & $-0,15^{\text {ns }}$ & $-0,52^{* *}$ & $-0,48^{* *}$ & $-0,15^{\mathrm{ns}}$ & $-0,05^{\mathrm{ns}}$ & $0,59^{* *}$ & $-0,54^{* *}$ \\
\hline DMS & 4,31 & 3,45 & 10,89 & 0,39 & 3,93 & 10,6 & 9,46 \\
\hline CV $(\%)$ & 16,41 & 5,76 & 10,13 & 11,75 & 17,82 & 7,98 & 5,92 \\
\hline Média & 12,47 & 27,12 & 53,42 & 1,26 & 6,75 & 49,45 & 27,26 \\
\hline
\end{tabular}

Médias seguidas por uma mesma letra na coluna não diferem entre si em nível de $5 \%$ de probabilidade pelo teste Tukey. ${ }^{* *}$ Significativo em nível de $1 \%$ de probabilidade pelo teste t. ${ }^{*}$ Significativo em nível de $5 \%$ de probabilidade pelo teste t. ${ }^{\text {ns }}$ Não significativo.

diâmetro das gotas pulverizadas. Dentre os diferentes produtos ensaiados, a solução contendo o adjuvante Poliflex apresentou o menor diâmetro quanto ao parâmetro $\mathrm{DV}_{0.5}$, e o maior valor da relação $\mathrm{Q} / \mathrm{M}$. Em contrapartida, o adjuvante Silwet proporcionou o maior valor de $\mathrm{DV}_{0,5}$ e o menor valor de $\mathrm{Q} / \mathrm{M}$. Este fato pode ser atribuído novamente, de acordo com CHAIM et al. (2002), ao tamanho das gotas, pois, quanto menor o diâmetro das gotas, mais facilmente ocorre o seu carregamento.

Na pulverização de agrotóxicos, o tamanho das gotas é um dos parâmetros mais importantes para o controle eficaz de pragas e doenças (YU et al., 2009). De uma forma geral, recomenda-se que as gotas pulverizadas sejam suficientemente grandes para não se perderem por evaporação, e pequenas o suficiente para fornecerem uma boa cobertura do alvo (NASCIMENTO et al., 2012). Ainda, o espectro de gotas deve ser o mais homogêneo possível, de tal forma que, quanto mais próximo de zero for o parâmetro Span, mais homogêneo é o espectro, aumentando as chances de atingir o alvo e reduzir perdas para o meio ambiente.

Outro quesito importantíssimo na aplicação de agrotóxicos é a determinação da porcentagem do volume que compõe o espectro de gotas. ARVIDSSON et al. (2011) afirmam que a porcentagem de gotas $<102 \mu \mathrm{m}$ é o melhor parâmetro para predizer o risco de deriva, quando comparado ao parâmetro $\mathrm{DV}_{0,5}$. De forma geral, o uso dos diferentes adjuvantes proporcionou aumento da porcentagem do volume de líquido para gotas de menores diâmetros na faixa de $11<\% \mathrm{~V}<30 \mu \mathrm{m}$ e, comparado à testemunha, reduziu a porcentagem de gotas das classes de maiores diâmetros de $31<\% \mathrm{~V}<50 \mu \mathrm{m}$ (Tabela 2 ). No presente ensaio, a grande quantidade de gotas formadas foi na faixa de 11 a $50 \mu \mathrm{m}$. Se, por um lado, gotas pequenas proporcionam maior cobertura do alvo e favorecem o carregamento das gotas, por outro, são altamente susceptíveis à evaporação e deriva.

$\mathrm{Na}$ avaliação da correlação de Person, verificou-se correlação positiva entre a relação Q/M e a condutividade elétrica, e correlação negativa entre a relação $\mathrm{Q} / \mathrm{M}$ e os parâmetros $\mathrm{DV}_{0,5}$ e $\mathrm{DV}_{0,9}$ (Tabela 1 e 2). A análise de correlação valida os resultados já demonstrados. O aumento da condutividade elétrica da solução e/ou a redução do diâmetro de gotas ocasionou o aumento da carga elétrica presente na gota. Observou-se também, que a viscosidade do líquido, no presente ensaio, não apresentou correlação com nenhuma variável.

Tendo em vista os resultados apresentados, o uso de adjuvantes na pulverização eletrostática pode melhorar a eficiência desta tecnologia, visto que, com o aumento da carga elétrica da gota, as forças de atração entre a gota e o alvo aumentam, consequentemente, maiores são as deposições de líquido no alvo. Contudo, nas condições analisadas, verificou-se que o diâmetro de gotas produzidas foi extremamente fina, e a adição dos adjuvantes reduziu ainda mais o tamanho das gotas, devendo haver, neste caso, atenção para as condições psicrométricas do ar, visando a minimizar os efeitos meteorológicos no momento da pulverização. 


\section{CONCLUSÃO}

Diante do relatado e de acordo com as condições experimentais estabelecidas, pode-se concluir que os adjuvantes melhoraram a eletrificação das gotas. Todos os produtos testados reduziram o diâmetro da mediana volumétrica. A carga elétrica na gota apresentou correlação positiva com a condutividade elétrica e negativa com o diâmetro das gotas.

\section{REFERÊNCIAS}

ARVIDSSON, T. et al. Spray drift as influenced by meteorological and technical factors. Pesticide Management Science, v.67, p.586-598, 2011. Disponível em: <http://onlinelibrary.wiley.com/ doi/10.1002/ps.2114/pdf>. Acesso em: 03 dez. 2013. doi: 10.1002/ ps. 2114 .

BUENO, M.R. et al. Estudo do espectro de gotas produzidas nas pulverizações aérea e terrestre na cultura da batata. Revista de Ciências Agrárias, v.54, n.3, p.225-234, 2011. Disponível em: <http:// www.periodicos.ufra.edu.br/index.php/ajaes/article/view/312/203>. Acesso em: 03 dez. 2013. doi:10.4322/rca.2012.018.

CHAIM, A. et al. Eficiência de deposição de agrotóxicos obtida com bocal eletrostático para pulverizador costal motorizado. Pesquisa Agropecuária Brasileira, v.37, n.4, p.497-501, 2002. Disponível em: <http://www.scielo.br/pdf/pab/v37n4/9083.pdf $>$. Acesso em: 03 dez. 2013.

CUNHA, J.P.A.R.; ALVES, G.S. Características físico-químicas de soluções aquosas com adjuvantes de uso agrícola. Interciência, v.34, n.9, p.655-659, 2009. Disponível em: $<$ http://www.scielo.org. ve/pdf/inci/v34n9/art12.pdf>. Acesso em: 03 dez. 2013.

CUNHA, J.P.A.R. et al. Efeito da temperatura nas características físico-químicas de soluções aquosas com adjuvantes de uso agrícola. Planta Daninha, v.28, n.3, p.665-672, 2010b. Disponível em: <http://www.scielo.br/pdf/pd/v28n3/24.pdf>. Acesso em: 03 dez. 2013. doi: 10.1590/S0100-83582010000300024.

CUNHA, J.P.A.R. et al. Espectro de gotas de pontas de pulverização com adjuvantes. Planta Daninha, v.28, p.1153-1158, 2010a. Disponível em: <http://www.scielo.br/pdf/pd/v28nspe/ a23v28nspe.pdf>. Acesso em: 03 dez. 2013. doi: 10.1590/S010083582010000500023 .

CUNHA, J.P.A.R.; PERES, T.C.M. Influência de pontas de pulverização e adjuvante no controle químico da ferrugem asiática da soja. Acta Scientiarum Agronomy, v.32, n.4, p.597-602, 2010. Disponível em: <http://www.scielo.br/pdf/asagr/v32n4/a05v32n4. pdf $>$. Acesso em: 03 dez. 2013. doi: 10.4025/actasciagron. v32i4.2502.

LAW, S.E. Electrostatic atomization and spraying. In et al. (Eds.). Handbook of electrostatic processes. New York: Marcel Dekker, 1995. p.413-440.

MASKI, D.; DURAIRAJ, D. Effects of electrode voltage, liquid flow rate, and liquid properties on spray chargeability of an airassisted electrostatic-induction spray-charging system. Journal of Electrostatics, v.68, p.152-158, 2010. Disponível em: <http:// www.sciencedirect.com/science/article/pii/S0304388609002332>. Acesso em: 03 dez. 2013. doi: 10.1016/j.elstat.2009.12.001

MOTA, A.A.B.; ANTUNIASSI, U.R. Influência de adjuvantes no espectro de gotas de ponta com indução de ar. Energia na Agricultura, v.28, n.1, p.1-5, 2013. Disponível em: <http:// energia.fca.unesp.br/index.php/energia/article/view/918/349>. Acesso em: 03 dez. 2013.

NASCIMENTO, A.B. et al. Deposição de glifosato e utilização de adjuvante para diferentes pontas de pulverização e horário de aplicação. Revista Brasileira de Tecnologia Aplicada nas Ciências Agrárias, v.5, n.2, p.105-116, 2012. Disponível em: $<$ http://revistas. unicentro.br/index.php/repaa/article/viewArticle/1672>. Acesso em: 03 dez. 2013. doi:10.5777/PAeT.V5.N2.08.

SASAKI, R.S. et al. Deposição e uniformidade de distribuição da calda de aplicação em plantas de café utilizando a pulverização eletrostática. Ciência Rural, v.43, n.9, p.16051609, 2013. Disponível em: <http://www.scielo.br/pdf/cr/v43n9/ a26713cr6928.pdf>. Acesso em: 03 dez. 2013.

YU, Y. et al. Evaporation and deposition coverage area of droplets containing insecticides and sprays additives on hydrophilic, hydrophobic, and crabapple leaf surfaces. Transactions of the ASABE, v.52, n.1, p.39-49, 2009. Disponível em: <http://naldc. nal.usda.gov/catalog/30444>. Acesso em: 03 dez. 2013.

ZHENG, J. et al. Advances in pesticide electrostatic spraying in China. Transaction of the ASAE, v.34, n.2, p.1-12, 2002. Disponível em: $<$ http://elibrary.asabe.org/abstract.asp?aid $=9304 \& \mathrm{t}=2 \&$ redir $=\&$ redirType $=>$. Acesso em: 03 dez. 2013. doi: 10.13031/2013.9304.

ZHOU, Y. et al. Development and application prospects of pneumatic electrostatic sprayer in orchard. Asian Agricultural Research, v.4, n.1, p.78-80, 2012. Disponível em: <http:// ageconsearch.umn.edu/bitstream/133110/2/18.PDF>. Acesso em: 03 dez. 2013. 